\title{
IONOSPHERIC EFFECTS OF TWO SOLAR FLARES AT THE MAXIMUM OF SOLAR CYCLE 23 AND AT THE MINIMUM OF SOLAR CYCLE 24
}

\author{
V.M. Smirnov \\ V.A. Kotelnikov Institute of Radio Engineering \\ and Electronics of RAS, \\ Fryazino, Russia,vsmirnov@ire.rssi.ru
}

\author{
E.V. Smirnova \\ V.A. Kotelnikov Institute of Radio Engineering \\ and Electronics of RAS, \\ Fryazino, Russia, evsmirnova58@mail.ru
}

\begin{abstract}
Using data from the GPS and GLONASS navigation satellite systems, we analyze the responses of the mid-latitude ionosphere to the extreme solar flares that occurred at the maximum of solar cycle 23 (October 28,2003$)$ and at the minimum of solar cycle 24 (September 6, 2017) during the same season at close solar zenith angles. To obtain the response, we use the rate of change of the total electron content, which is practically independent of characteristics of equipment and determined only by parameters of a propagation medium (the ionosphere in our case). The ionospheric
\end{abstract}

response is shown to be almost independent of the total duration of the flare. In both cases, the duration of the main response at a level of 0.5 is about $1.5-2 \mathrm{~min}$, whereas the total duration of the response is about 10 min and almost independent of solar flare power.

Keywords: ionosphere, solar flares, navigation satellite systems, radio translucence method, electron content.

\section{INTRODUCTION}

The solar flare is a sudden and rapid release of a huge amount of energy in the upper chromosphere or lower corona of the Sun. The generated electromagnetic emission in the wavelength range from $\lambda \sim 0.5-8 \AA$ to $\lambda \sim 1 \mathrm{~m}$ [Avakyan et al., 1994; Mitra, 1977] causes strong disturbances in various layers of Earth's ionosphere, and consequently a significant change in parameters of nearEarth space for monitoring of which the mid-orbital navigation satellite systems GPS and GLONASS are now being actively used.

The use of data from these networks has made it possible to take a new step in studying the impact of solar flares on Earth's ionosphere [Afraimovich, Perevalova, 2006; Afraimovich, 2000]. The method of monitoring ionospheric total electron content (TEC) disturbances of natural and anthropogenic origin, which is based on phase measurements of parameters of satellite navigation systems, is extensively applied nowadays to the detailed analysis of solar activity effects. For example, Kunitsyn et al. [2015] have analyzed severe solar flares of solar cycle 23, including the October 28, 2003 flare. The ionospheric disturbances generated by this flare have been examined in [Smirnov Smirnov, 2005, 2014; Zhang, Xiao, 2005], where similar dependences for the ionospheric response were obtained.

Le et al. [2013] have analyzed about 100 X-class flares, which occurred in solar cycle 23 from 1999 to 2006. They examined the influence of X-rays and UV emission on TEC variations depending on the solar zenith angle and season of flare occurrence, and also the possibility of using the ionospheric response to determine the UV emission intensity for flares of class X6 and lower. Liu et al. [2004, 2011] briefly review works devoted to the study of ionospheric disturbances generated by solar flares of solar cycles 23 and 24 .
The above works generally study the dependence of the ionospheric response maximum on the power of flares and their position on the solar disk, on solar zenith angle, season of their occurrence, and solar cycle.

Our purpose here is to analyze the response of the mid-latitude ionosphere to the impact of two extreme solar flares that occurred at the maximum of solar cycle 23 and at the minimum of solar cycle 24 in the fall season at close zenith angles and sunspot positions on the solar disk.

\section{SOLAR FLARES OF CYCLES 23 AND 24}

One of the most powerful solar flares occurred on October 28, 2003, at the maximum of solar cycle 23. It is described in detail in the literature [Belov et al., 2004; Veselovsky et al., 2004]. A special issue of the journal Geomagnetism and Aeronomy (2005, volume 45, number 1) is dedicated to the extreme solar events that took place in October-November 2003 and their associated abnormal processes in Earth's magnetosphere and ionosphere. The flare began at 09:51 UT, peaked at 11:10 UT, and ended at 11:24 UT. So it lasted for $1 \mathrm{hr} 33 \mathrm{~min}$.

Figure 1 shows solar activity for October 28, 2003. An unusually strong increase in X-ray intensity began at $\sim 11$ UT. It was also accompanied by an increase in UV emission intensity, which caused additional ionization in the ionospheric F-region. The October 28, 2003 heliophysical event occurred in the active region when a group of sunspots was at the central meridian of the Sun. The particle flux was directed immediately to Earth.

The solar flare that was the largest in the past 12 years was recorded by the Solar Dynamics Observatory (SDO) in the 2673 active region on September 6, 2017 at 12:02 UT. According to NOAA data, the flare began at 11:53 UT, peaked at 12:02 UT, and ended at 12:10 UT; so, it lasted for $17 \mathrm{~min}$. The X9.3 flare occurred due to the interaction of two sunspot groups, which were the 


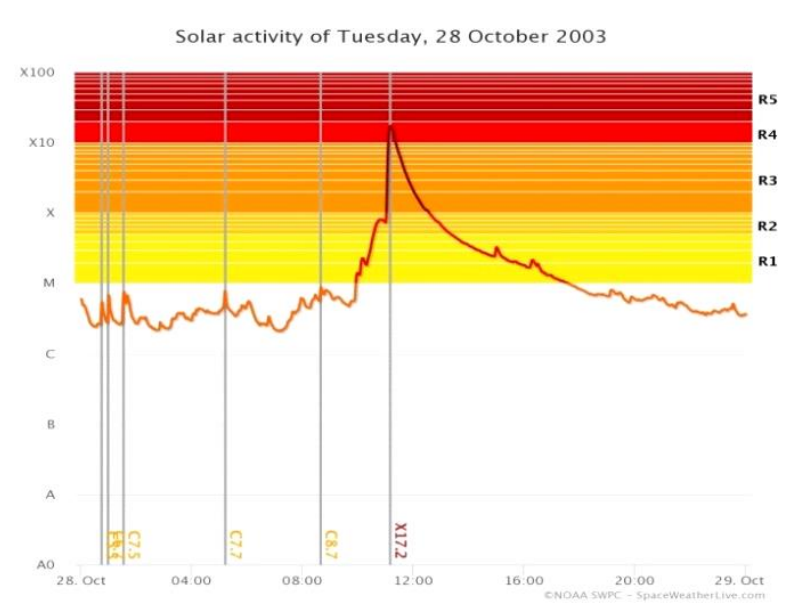

Figure 1. Solar activity on October 28, 2003 [https://www.spaceweatherlive.com/en/archive/2003/10/28/xray]

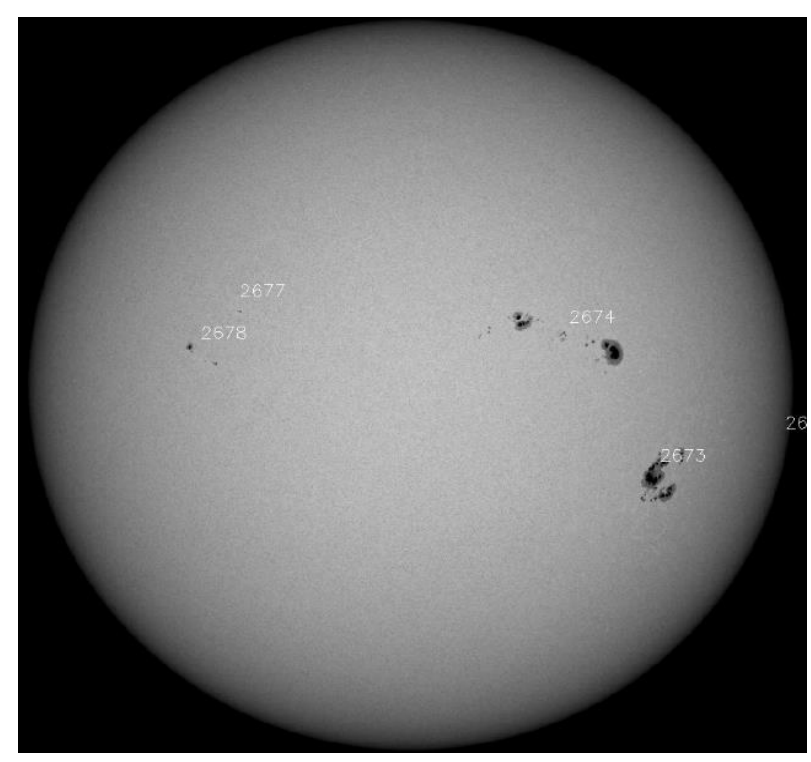

September 6, 2017

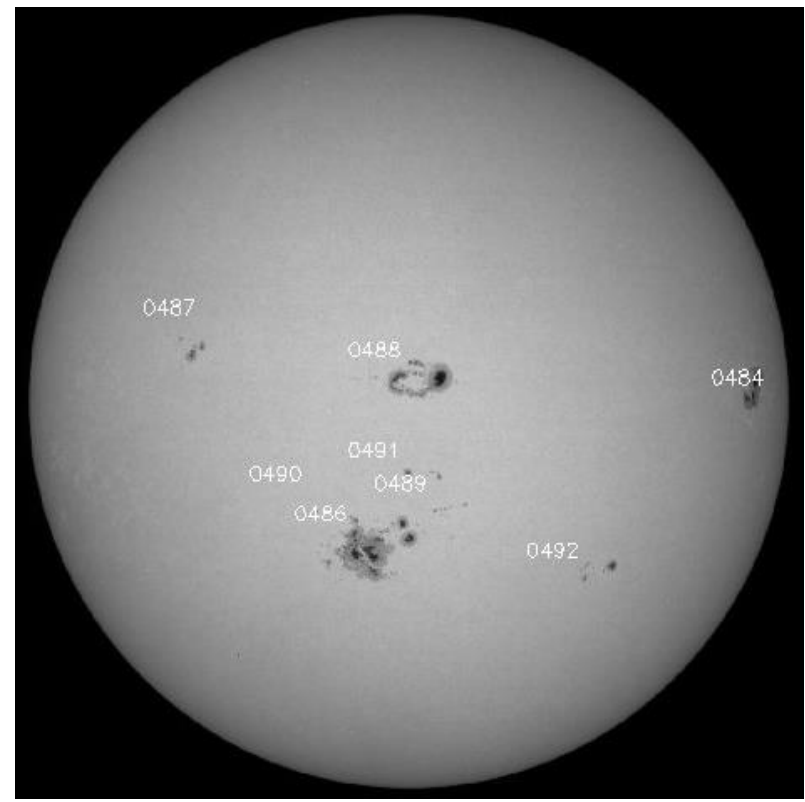

October 28, 2003

Figure 2. Sunspots on the visible solar disk on October 28, 2003 and September 6, 2017 [https://www.spaceweatherlive.com/en/reports/sunspot-report] largest within the last few years. Figure 2 shows how they looked like on the visible solar disk on September 6, 2017. By comparison, this Figure also presents the visible solar disk for October 28, 2003. The Figures are adopted from [https://www.spaceweatherlive.com]. It should be noted that the sunspots observed on the Sun on October 28, 2003 and September 6, 2017 were approximately at the same latitude. This is important because, as is shown in [Leonovich et al., 2010] by the example of the July 14, 2000 solar flare, the TEC value depends on the flare position on the solar disk.

According to data from the Solar X-ray Astronomy Laboratory of LPI RAS, the September 6, 2017 flare is one of the most powerful explosions the Sun is capable of producing. For 20 years of observations, only five severe flares have been recorded (the last one (X17.0) occurred in November 2005). These events generally happened at the solar maximum, but this flare occurred at the solar minimum, and therein lies its uniqueness. Notice that the September 6, 2017 flare, as well as the October 28, 2003 flare, occurred in the geoeffective region near the Sun-Earth line, from which the Sun has the strongest effect on our planet.

The sunspot activity after the explosion was X9.3, which corresponds to the highest level. Figure 3 depicts solar activity for September 6, 2017.

Figure 4 shows the X-ray intensity in the range 1-8 and 0.5-4 $\AA$, observed with instruments on GOES satellites on September 6, 2017 [https://www.swpc.noaa.gov/products/ goes-x-ray-flux]. The flare was observed only by foreign space observatories.

\section{ANALYSIS OF THE RESULTS}

Electromagnetic emission of a wide spectral range causes strong disturbances in various regions of Earth's ionosphere. During a flare, the Sun is a powerful source of X-rays and ultraviolet emission. An increase in ionizing radiation intensity, observed during solar flares, immediately causes an increase in electron density and TEC in the ionosphere. To determine ionospheric parameters, we have used data from the global navigation satellite systems GPS and GLONASS. Due to dualfrequency measurements in the L-band (about 1.2 and 1.6 $\mathrm{GHz}$ ), the electron content can be monitored using hundreds of permanent ground navigation signal receivers

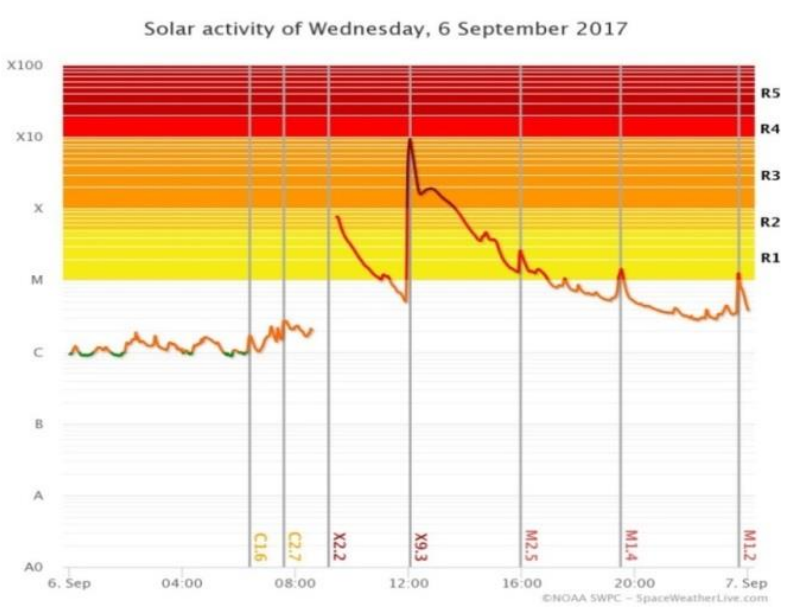

Figure 3. Solar activity on September 6, 2017 


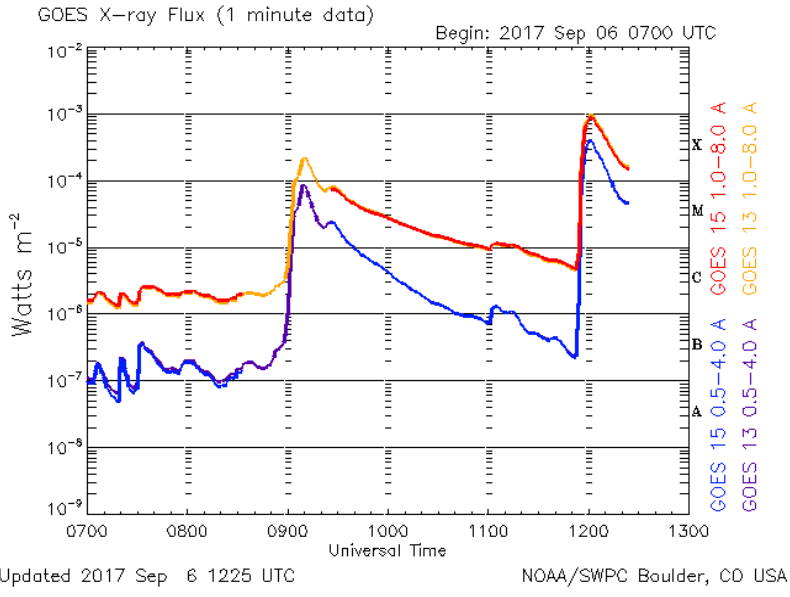

Figure 4. X-ray intensity in ranges $1-8$ and $0.5-4 \AA$ on September 6, 2017. The data were taken from NOAA's Space Weather Prediction Center website [https://www.swpc.noaa. gov/products/goes-X-ray-flux]

with unprecedented temporal and spatial resolution. The global navigation satellite systems allow not only 24hour monitoring of the ionosphere at a certain point, but also allow us to use the same satellite for monitoring the ionosphere at different points of Earth's surface, which are spaced apart by thousands of kilometers. One satellite provides information with the same degree of measurement accuracy if there are similar positioning receivers at ground stations. Ionospheric parameters were identified by the radio translucence method along the satellite-Earth path. Aspects of their application are thoroughly discussed in [Andrianov, Smirnov, 1993; Andrianov et al., 1996, 2000; Smirnov, 2001]. To determine the ionospheric parameters, we have used phase measurements made at elevation angles greater than $15^{\circ}$.

Neglecting the influence of collisions and geomagnetic field on the refractive index of the ionosphere, the formula for calculating TEC along the satellite-receiver ray path can be represented as the following simple relation [Kolosov et al., 1969; Hofmann-Wellenhof et al., 1992]

$$
\operatorname{TEC}(t)=\int_{l} N_{\mathrm{e}}(l) d l=K\left(\lambda_{1} \Phi_{1}(t)-\lambda_{2} \Phi_{2}(t)\right)+\text { const },
$$

where $K=\frac{1}{40.308} \frac{f_{1}^{2} f_{2}^{2}}{f_{1}^{2}-f_{2}^{2}}, \quad f_{1}=1575.42, \quad f_{2}=1227.6$ $\mathrm{MHz}$ are the operating frequency of GPS; for GLONASS $f_{1}=\left(1602+m \Delta f_{1}\right) \quad \mathrm{MHz}$ and $f_{2}=$ $\left(1246+m \Delta f_{2}\right) \mathrm{MHz}, m=0, \ldots, 24$ are numbers of characters of carrier frequencies of navigation radio signals, $\Delta f_{1}=0.5625 \mathrm{MHz}, \Delta f_{2}=0.4375 \mathrm{MHz}, \Phi_{1,2}(t)$ is the phase , $\lambda_{1}$ and $\lambda_{2}$ are the wavelengths of corresponding emission frequencies.

The presence of the second term in this formula makes it impossible to determine the absolute TEC directly from phase measurements. However, solving certain problems requires knowing TEC variations rather than its absolute value. The rate of TEC variations or its increment in the observed time interval is the ionospheric parameter most sensitive to external factors. Values of this parameter are almost independent of characteristics of satellite signal transmitters and receivers, they are determined only by the propagation medium — the ionosphere in this case.

The rate of TEC variations is calculated from the formula

$$
\begin{aligned}
& \operatorname{DTEC}(t)= \\
& =K \frac{\left\{\lambda_{1}\left[\Phi_{1}(T+t)-\Phi_{1}(t)\right]-\lambda_{2}\left[\Phi_{2}(T+t)-\Phi_{2}(t)\right]\right\}}{T},
\end{aligned}
$$

where $T$ is the observation time interval. For organizational IGS stations, the observation time interval is usually $30 \mathrm{~s}$.

The DTEC $(t)$ parameter was calculated directly from measurements of $\Phi_{1,2}(t)$ without using any smoothing procedures and digital filters. For ease of comparison, the calculation results are normalized to maximum $D T E C$ values. In general, the shape of the ionospheric response obtained at monitoring stations, is practically identical: all characteristic features of DTEC variations are observed at the same time points.

Figures 5 and 6 depict the ionospheric responses to the October 28, 2003 and September 6, 2017 solar flares. These Figures present the results for the MDVJ station (Mendeleevo, the Moscow Region), obtained for two satellites. Left panels show the results for the entire observation session; right panels depict in more detail a fragment with the ionospheric response during the solar flare. By comparison, the same fragments are shown in a larger size in Figures 5 and 6 at the bottom. It is clear that the characteristic details of the responses are almost identical for each of the solar flares.

For the October 28, 2003 solar flare, the duration of the main response at a level of 0.5 is about $2 \mathrm{~min}$, the total duration of the response is about $10 \mathrm{~min}$, with the total duration of the solar flare being $1 \mathrm{hr} 33 \mathrm{~min}$. $\operatorname{DTEC}(t)$ has two distinct maxima. The first maximum virtually corresponds to the time of the solar flare onset and is recorded $8 \mathrm{~min}$ before the maximum emission intensity in the optical range.

For September 6, 2017 solar flare, the duration of the main response at a level of 0.5 is about $1.5 \mathrm{~min}$, the total duration of the response is about $10 \mathrm{~min}$, with the total duration of the solar flare being $17 \mathrm{~min}$. DTEC $(t)$ has two pronounced maxima, the first maximum virtually corresponds to the time of the solar flare onset and is also recorded $8 \mathrm{~min}$ before the maximum emission intensity in the optical range.

The shape of the second maximum of the response, observed at 11:58 UT (718 $\mathrm{min})$, is sufficiently close to the shape of the first response (see Figure 7). A cause for another maximum of the response could be the second flare masked with the first one. The analysis of the September 6, 2017 ionosphere response allowed us to identify a weaker flare (X2.2), which occurred at 09:10 UT. It started at 08:57 UT, peaked at 09:10 UT, and ended at 09:17 UT. The total duration of the X2.2 flare was $20 \mathrm{~min}$. The response in this case also has two maxima spaced apart in time by 4 min as in the X9.3 flare. While durations of the main ionospheric responses are approximately the same, their shapes differ substantially from each other (see Figures 5 and 6). If for the flare at the maximum of solar cycle 23 the response has one pronounced double maximum, the flare at the minimum has two distinct maxima with a sharp 


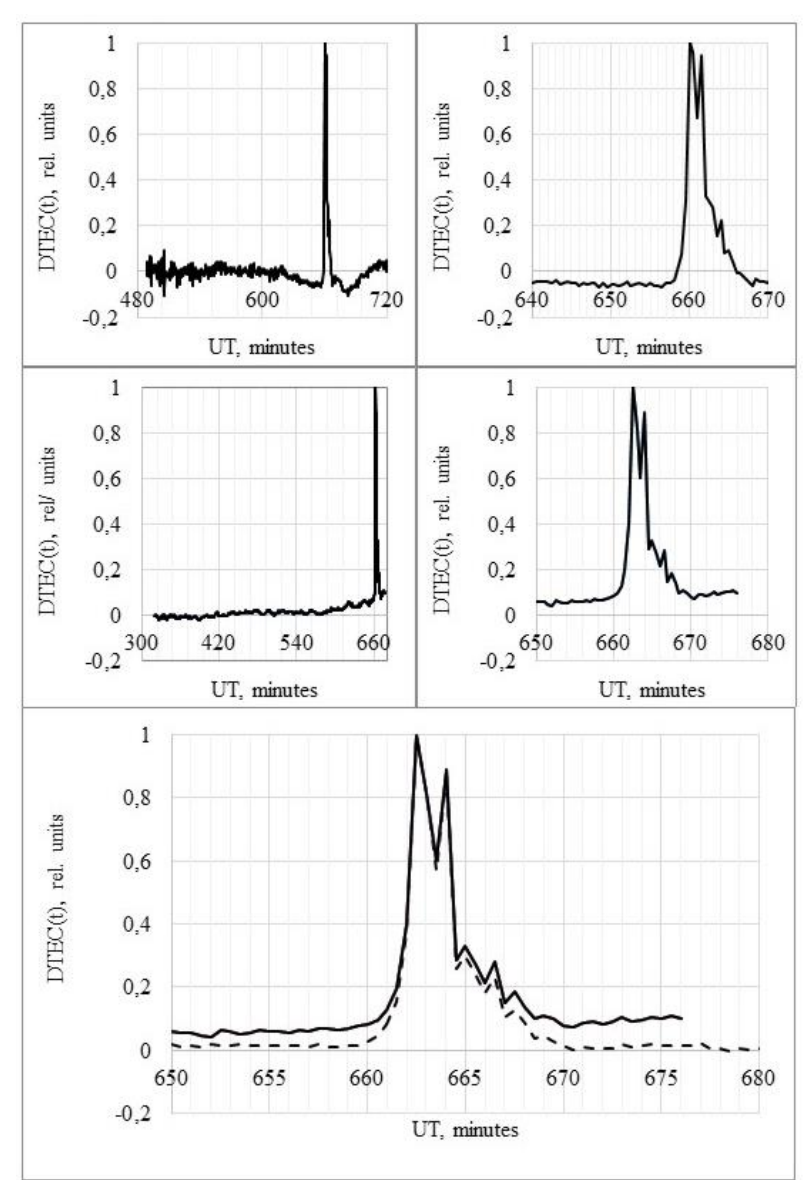

Figure 5. Ionospheric response to the October 28, 2003 solar flare for GPS satellites No. 26 (top panels) and No. 29 (middle panels). Bottom panel: dashed line is GPS No. 26; solid line is GPS No. 29

drop between them, which is likely to be due to the time dynamics of the electromagnetic emission of the flares.

The two maxima in the September 6, 2017 X2.2 and X9.3 solar flares might have been caused either by the occurrence of less severe flares accompanied by them and leading to their enhancement, or by peculiarities of their generation during solar minimum.

\section{CONCLUSION}

The paper has examined the impact of two extreme solar flares, which occurred at the maximum of solar cycle 23 and at the minimum of solar cycle 24, on ionospheric conditions and has analyzed shapes of the ionospheric response to these heliophysical events. These flares took place in the fall period almost at the same time of day near the vicinity of the Sun-Earth line, from which the Sun has the strongest effect on our planet.

To determine ionospheric parameters by the radio translucence method along the satellite-Earth path, we have used data from the global navigation satellite systems GPS and GLONASS. We have shown that in both the cases the duration of the main response at the 0.5 level is $1.5-2 \mathrm{~min}$, with the total duration of the response being $\sim 10 \mathrm{~min}$ and independent of solar flare importance. While durations of the ionospheric responses are roughly the same, their shapes differ significantly. The second response on September 6, 2017 might have

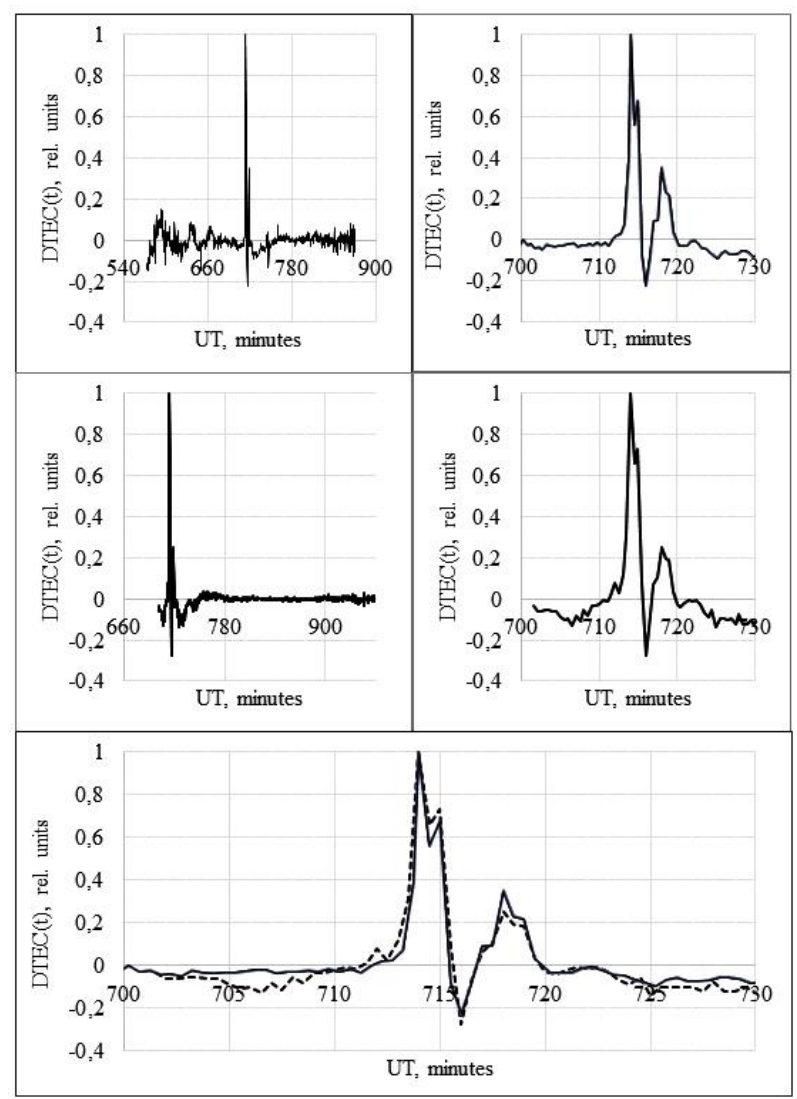

Figure 6. Ionospheric response to the September 6, 2017 solar flare for GPS satellites No. 10 (top panels) and No. 32 (middle panels). Bottom panel: dashed line is GPS No. 32; solid line is GPS No. 10
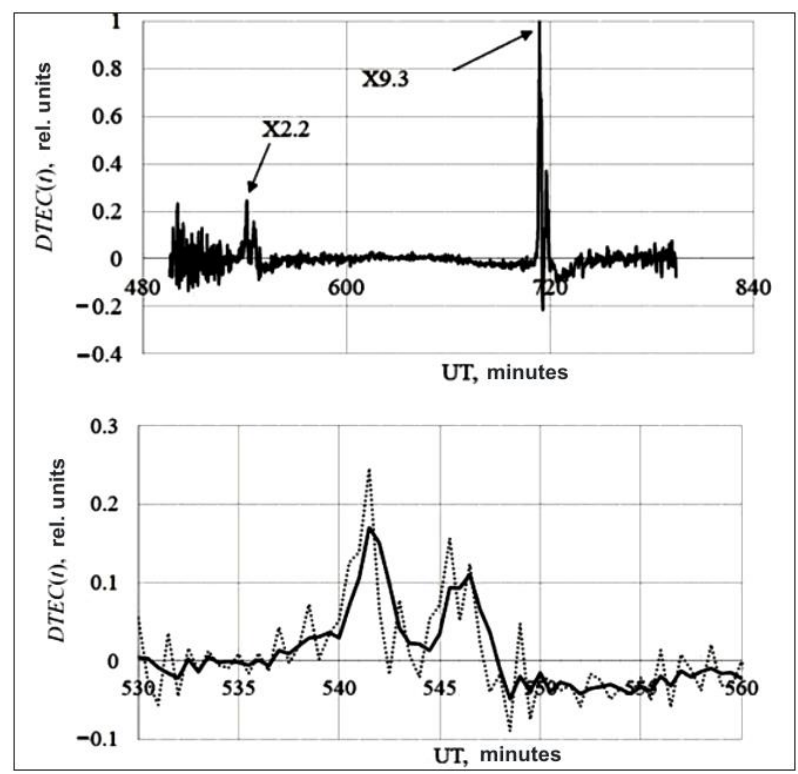

Figure 7. Ionospheric response to the September 6, 2017 solar flare $(a)$ and a fragment with the response to the $\mathrm{X} 2.2$ flare $(b$, solid line is the linear filtering)

been caused by the second flare, which was masked with the first flare.

This work was carried out within the framework of the state task and partially supported by RAS Presidium Basic Research Program No. 12 "Problems of origin and evolution of the Universe, solved using methods of 
ground-based and satellite observations".

\section{REFERENCES}

Afraimovich E.L. GPS global detection of the ionospheric response to solar flares. Radio Sci. 2000, vol. 35, pp. 14171424. DOI: $10.1029 / 2000 R S 002340$.

Afraimovich E.L., Perevalova N.P. GPS-monitoring verkhnei atmosfery Zemli [GPS Monitoring of the Earth's Upper Atmosphere]. Irkutsk, 2006, 480 p. (In Russian).

Andrianov V.A., Smirnov V.M. Determining a height profile of Earth's ionosphere electron density from double-frequency measurements of satellite radio signals. Radiotekhnika $i$ elekctronika [J. of Communications Technology and Electronics] 1993, vol. 38, no. 7, pp. 1326-1335. (In Russian).

Andrianov V.A., Armand N.A., Mosin E.L., Smirnov V.M Results of using NAVSTAR navigation system for Earth's ionosphere monitoring. Issledovanie Zemli iz kosmoca [Izvestiya, Atmospheric and Oceanic Physics]. 1996, no. 2, p. 10. (In Russian).

Andrianov V.A., Mosin E.L., Smirnov V.M. Determining regular variations of the Earth's ionosphere F2 region from measurements of signal parameters of navigation satellites. Issledovanie Zemli iz kosmoca [Izvestiya, Atmospheric and Oceanic Physics]. 2000, no. 1, p. 15. (In Russian).

Avakyan S.V., Vdovin A.I., Pustarnakov V.F. Ioniziruyushchee $i$ pronikayushchee izluchenie $v$ okolozemnom kosmicheskom prostranstve [Ionizing and Penetrating Radiation in the Near-Earth Space]. Saint Petersburg, Gidrometeoizdat Publ., 1994, 501 p. (In Russian).

Belov A.V., Gaidash S.P., Ivanov K.G., Kanonidi Kh.D. Unusually high geomagnetic activity in 2003. CosmicRes. 2004, vol. 42 , the no. 6, pp. 541-550. DOI: 10.1007/s10604-005-0001-0.

Hofmann-Wellenhof B., Lichtenegger H., Collins J. Global Positioning System: Theory and Practice. 2nd ed., New York, Springer-Verlag, 1992, $347 \mathrm{p}$.

Kolosov M.A., Armand N.A., Yakovlev O.I. Rasprostranenie radiovoln pri kosmicheskoi svyazi [Radio Wave Propagation in Space Communication]. Moscow, Svyaz' Publ., 1969, 155 p. (In Russian).

Kunitsyn V.E., Nazarenko M.O., Nesterova I.A., Padokhin A.M. Solar flare forcing on ionization of upper atmosphere. Comparative study of several major X-class events of 23rd and $24^{\text {th }}$ solar cycles. Moscow University Phys. Bull. 2015, vol. 70, no. 4, pp. 312-318. DOI: $10.3103 / \mathrm{S} 0027134915040128$.

Le H., Liu L., Chen Y., Wan W. Statistical analysis of ionospheric responses to solar flares in the solar cycle 23. J.Geophys. Res. 2013, vol. 118, pp. 576-582. DOI: 10.1029/2012JA017934.

Leonovich L.A., Taschilin A.V., Portnyagina O.Y. Dependence of the ionospheric response on the solar flare parameters based on the theoretical modeling and GPS data. Geomagn. Aeron. 2010, vol. 50, pp. 201-210. DOI: 10.1134/ S0016793210020076.

Liu L., Wan W., Chen Y., Le H. Solar activity effects on the ionosphere: a brief review. Chinese Sci. Bull. 2011, vol. 56, no.12, pp. 1202-1211. DOI: 10.1007/s11434-010-4226-9.

Liu J.-Y., Lin C.H., Tsai H.F., Liou Y.A. Ionospheric solar flare effects monitored by the ground-based GPS receivers: Theory and observations. J. Geophys. Res. 2004, vol. 109, A01307. DOI: 10.1029/2003JA009931.

Mitra A. Vozdeistvie solnechnykh vspyshek na ionosferu Zemli [Effects of Solar Flares on Earth's Ionosphere]. Moscow, Mir Publ., 1977. 370 p. (In Russian). English edition: Mitra A.P. Ionospheric Effects of Solar Flares. Dordrecht, D. Reidel Publ. Co., 1974, 305 p.

Smirnov V.M. Solution of the inverse problem of electromagnetic transmission probing of the Earth ionosphere by gradient methods. J. Communications Technology and Electronics. 2001, vol. 46, no. 1, pp. 41-45.
Smirnov V.M., Smirnova E.V. Time variations in the ionosphere during the solar flare of October 28, 2003, according to data of the GPS network. Geomagn. Aeron. 2005, vol. 4 no. 1 , pp. $122-128$.

Smirnov V.M., Smirnova E.V. Ionospheric response to the extreme solar burst of October 28, 2003. Geomagn. Aeron. 2014, vol. 54, no. 1, pp. 87-93. DOI: 10.1134/ S0016793214010149.

Veselovsky I.S., Panasyuk M.I., Bogomolov A.V., Denisov Yu.I., Dmitriev A.V., Zhukov A.N., Zeldovich M.A., et al. Solar and heliospheric phenomena in October-November: causes and effects. Cosmic Res. 2004, vol. 42, no. 5, pp. 435488.

Zhang D.H., Xiao Z. Study of ionospheric response to the 4B flare on 28 October 2003 using International GPS Service network data. J.Geophys. Res. 2005, vol. 110, A03307. DOI: 10.1029/2004JA010738.

URL: https://www.spaceweatherlive.com (accepted November 10, 2018).

URL: https://www.spaceweatherlive.com/en/archive/2003/10/ 28/xray (accepted November 10, 2018).

URL: https://www.spaceweatherlive.com/en/reports/sunspotreport (accepted November 10, 2018).

URL: https://www.swpc.noaa.gov/products/goes-x-ray-flux (accepted September 7, 2017).

How to cite this article

Smirnov V.M., Smirnov E.V. Ionospheric effects of two solar flares at the maximum of solar cycle 23 and at the minimum of solar cycle 24. Solar-Terrestrial Physics. 2019. Vol. 5, Iss. 2. P. 76-80. DOI: $10.12737 /$ stp-52201911. 